\title{
The Effect of Primary User Traffic on the Performance of Robust and Conventional Goodness of Fit (GOF) Test based Spectrum Sensing Methods in Cognitive Radio
}

\begin{tabular}{|c|c|c|}
\hline \multicolumn{3}{|c|}{ Timur DÜZENLİ*1, Olcay AKAY ${ }^{2}$} \\
\hline $\begin{array}{l}{ }^{1} \text { Amasya University, Technolo } \\
{ }^{2} \text { Dokuz Eylül University, Engin }\end{array}$ & $\begin{array}{l}\text { ulty, Department of Electrical ar } \\
\text { Faculty, Department of Electricc }\end{array}$ & $\begin{array}{l}\text { tronics Engineering, Amasya, TURKEY } \\
\text { Electronics Engineering, Izmir, TURKEY }\end{array}$ \\
\hline Başvuru/Received: 08/10/2017 & Kabul/Accepted: 01/12/2017 & Son Versiyon/Final Version: 26/12/2017 \\
\hline
\end{tabular}

\begin{abstract}
In cognitive radio, users of the spectrum are grouped as primary and secondary users. Primary users are license holders and a certain interval of band is assigned to them. On the other hand, secondary users determine the idle frequency intervals which are not used by primary users. To determine whether a spectrum band is idle or in use, various spectrum sensing techniques have been proposed in the literature. In the studies on goodness of fit (GOF) tests, it is assumed that primary user does not change its status during spectrum analysis. However, when the duration of analysis is long, the status of primary user may change. In this study, effect of the primary user traffic on the sensing performance of GOF test based spectrum sensing methods is investigated. According to simulation results, it has been observed that changes in the primary user status adversely affect the sensing performance.
\end{abstract}

\section{Key Words}

“Cognitive Radio, Primary User, Secondary User, Spectrum Sensing, Goodness of Fit Test, Primary User Traffic" 


\section{INTRODUCTION}

Goodness of fit (GOF) tests are considered as nonparametric spectrum sensing methods in the cognitive radio (CR) literature (Wang et al., 2009). They are also treated as blind spectrum sensing techniques since they do not need a priori knowledge of the primary user (PU) signal.

It is possible to improve performance of GOF tests by extending the length of the sensing period. However, use of longer sensing periods may also increase the possibility of having a dynamic PU which changes its status during the sensing period. Therefore, possible PU status changes (also called PU traffic) in the channel should also be taken into consideration by a CR. However, a great majority of the works on spectrum sensing based on GOF testing assumes that the PU does not change its status during the sensing period (Wang et al., 2009), (Rostami et al., 2012), (Lei et al., 2011). Hence, in this study, we investigate performances of GOF tests for detecting dynamic PUs under the existence of PU traffic.

The rest of the paper is organized as follows: The system model used in this study is introduced in Section 2. In Section 3, widely used GOF tests for spectrum sensing are presented. Simulation results are considered in Section 4, and finally, conclusions are given in Section 5.

\section{SYSTEM MODEL}

In the studies on spectrum sensing, the following system model is commonly used (Yücek and Arslan, 2009), (Düzenli and Akay, 2013)

$$
H_{0}: x[n]=w[n], \quad n=1,2, \ldots, N
$$

$H_{1}: x[n]=s[n]+w[n], \quad n=1,2, \ldots, N$

where $w[n]$ denotes samples of white Gaussian noise (WGN) distributed according to $\quad\left(0, \sigma^{2}\right), s[n]$ is the PU signal, and $N$ is the number of total samples observed during the sensing period.

In Eq. (1), it is assumed that there is no PU status change during the sensing period. If there is a status change of the PU in the channel, then the model given in Eq. (1) can be modified as

$$
\begin{aligned}
& H_{0}: x[n]=\left\{\begin{array}{cc}
s[n]+w[n], & n=1, \ldots, i_{1} \\
w[n], & n=i_{1}+1, \ldots N
\end{array}\right. \\
& H_{1}: x[n]=\left\{\begin{array}{cc}
w[n], & n=1, \ldots, j_{1} \\
s[n]+w[n], & n=j_{1}+1, \ldots N
\end{array}\right.
\end{aligned}
$$

where $i_{1}$ and $j_{1}$ are the PU status change points under $H_{0}$ and $H_{1}$, respectively.

In the same way, the system model for two status changes of the PU can be written as

$$
H_{0}: x[n]=\left\{\begin{aligned}
w[n], & n=1, \ldots, i_{1} \\
s[n]+w[n], & n=i_{1}+1, \ldots, i_{2} \\
w[n], & n=i_{2}+1, \ldots, N
\end{aligned}\right.
$$


$H_{1}: x[n]=\left\{\begin{array}{cc}s[n]+w[n], & n=1, \ldots, j_{1} \\ w[n], & n=j_{1}+1, \ldots, j_{2} \\ s[n]+w[n], & n=j_{2}+1, \ldots, N\end{array}\right.$

where $i_{1}$ and $i_{2}$ are the first and second status change points of the PU under hypothesis $H_{0}$, respectively. Similarly, $j_{1}$ and $j_{2}$ are the first and second status change points of the PU under hypothesis $H_{1}$.

The model in Eq. (3) can be extended for more than two PU status changes by assuming that hypothesis $H_{0}$ ends with noise-only samples and hypothesis $H_{1}$ ends with noise-corrupted signal samples (Düzenli and Akay, 2016).

It can be stated that hypothesis $H_{0}$ corresponds to the absence of the PU in the channel (null hypothesis), and oppositely, hypothesis $H_{1}$ is the case where PU is in the channel (alternative hypothesis). If $H_{1}$ is decided under hypothesis $H_{1}$, then it leads to probability of detection, $P_{D}$. That is; probability of deciding that there is PU signal in the channel. Thus, PU actually exists and is correctly detected. If $H_{1}$ is decided while $H_{0}$ is observed, then it refers to probability of false alarm, $P_{F A}$, which indicates deciding that the PU signal exists when there is actually no PU communication. Thus, error of false alarm leads to inefficient use of the spectrum.

$P_{D}$ and $P_{F A}$ can be expressed mathematically using the following definitions

$P_{D}=P\left(T_{\mathrm{x}}>\gamma_{\mathrm{x}} \mid H_{1}\right)$

$P_{F A}=P\left(T_{\mathrm{x}}>\gamma_{\mathrm{x}} \mid H_{0}\right)$

where $T_{\mathrm{x}}$ is the test statistic and $\gamma_{\mathrm{x}}$ is the decision threshold value for the test statistic. $P_{F A}$ should be kept as small as possible in order to prevent underutilization of transmission opportunities. The decision threshold $\gamma_{\mathrm{x}}$ can be selected for finding an optimum balance between $P_{D}$ and $P_{F A}$.

\section{GOF Tests for Spectrum Sensing}

In GOF testing, the main goal is to determine if the observed data come from a particular known distribution or not. This testing scheme can be adapted to spectrum sensing in CR. Using GOF testing, the binary hypothesis testing problem for spectrum sensing can be formulated as (Zhang, 2002)

$$
\begin{aligned}
& H_{0}: F_{N}(x)=F_{0}(x) \\
& H_{1}: F_{N}(x) \neq F_{0}(x) .
\end{aligned}
$$

In Eq. (6), $F_{0}(x)$ is a known cumulative distribution function (CDF) which is assumed to be present under the null hypothesis, $H_{0}$. 
Assuming a Gaussian communication channel as in (Wang et al., 2009), (Rostami et al., 2012), (Lei et al., 2011), $F_{0}(x)$ can be taken to be equal to the CDF of zero-mean Gaussian (normal) distribution, $\quad\left(0, \sigma^{2}\right)$, with variance $\sigma^{2} . F_{N}(x)$ corresponds to the empirical CDF which is calculated as (Rostami et al., 2012)

$F_{N}(x)=\frac{\left|i: x_{i} \leq x, 1 \leq i \leq N\right|}{N}$

where $|$.$| denotes cardinality, x_{i}$ is the $i^{\text {th }}$ sample of the observed data vector $\mathbf{x}$, and $N$ represents the number of observed samples. Without loss of generality, the elements of $\mathbf{x}$ are assumed to be real-valued.

There are several GOF tests proposed in the literature. In this paper, the following widely used GOF tests are considered:

- Anderson - Darling (AD) (Wang et al., 2009), (Rostami et al., 2012), (Lei et al., 2011), (Zhang, 2002), (N.-Thanh et al., 2012), (Arshad and Moessner, 2013)

- Kolmogorov - Smirnov (KS) test (Lei et al., 2011),

- Cramer - von Misses (CM) test (Lei et al., 2011),

- $\quad$ Log-Likelihood Ratio (LLR) GOF tests (Zhang, 2002),

- Order statistics (OS) (Rostami et al., 2012).

\subsection{Anderson-Darling (AD) Test}

In the $\mathrm{AD}$ test, it is aimed to measure the distance between the observed data and the known noise distribution. If the result of this measurement is smaller than a certain threshold, then $H_{0}$ is decided as the valid hypothesis.

The AD decision statistic is given as (Arshad and Moessner, 2013)

$T_{A D}=N \int_{-\infty}^{\infty}\left[F_{N}(x)-F_{0}(x)\right]^{2} \psi\left(F_{0}(x)\right) d F_{0}(x)$

where $\psi($.$) is a weight function defined as \psi(u)=[u(1-u)]^{-1}$ for the interval $0 \leq u \leq 1$.

It is possible to simplify the AD test statistic in Eq. (8) by performing the integral. Then, $T_{A D}$ can be obtained as (Arshad and Moessner, 2013)

$T_{A D}=-\frac{N^{2}+\sum_{i=1}^{N}(2 i-1)\left(\ln z_{i}+\ln \left(1-z_{N+1-i}\right)\right)}{N}$

where $z_{i}=F_{0}\left(x_{i}\right)$.

\subsection{Kolmogorov-Smirnov (KS) Test}

As in the AD test, the KS test aims to obtain how far the empirical CDF of the received signal is from the known CDF of the noise. It is also possible to interpret the KS test as measuring "graphically the maximum vertical distance between the two distributions, $F_{N}(x)$ and $F_{0}(x)$ "(Arshad and Moessner, 2013). 
Accordingly, the KS decision statistic is defined as (Lei et al., 2011)

$T_{K S}=\max \left|F_{N}(x)-F_{0}(x)\right|$

where $F_{N}(x)$ is the empirical CDF as defined in Eq. (7). According to Eq. (10), the test statistic $T_{K S}$ converges to zero under hypothesis $H_{0}$.

\subsection{Cramer-von Misses (CM) Test}

$\mathrm{n}$ the CM test, the distance between $F_{N}(x)$ and $F_{0}(x)$ is defined as (Lei et al., 2011), (Stephens, 1974)

$T_{C M}=N \int_{-\infty}^{+\infty}\left[F_{N}(x)-F_{0}(x)\right]^{2} d F_{0}(x)$

The CM test statistic can be simplified as (Lei et al., 2011)

$T_{C M}=\sum_{i=1}^{N}\left[z_{i}-\frac{(2 i-1)}{2 N}\right]^{2}+\left(\frac{1}{12 N}\right)$

where $z_{i}=F_{0}\left(x_{i}\right)$.

\subsection{Log-Likelihood Ratio (LLR) GOF tests}

In GOF testing, it is possible to derive the test statistics by two basic equations which are given as (Zhang, 2002)

$$
\begin{aligned}
& Z=\int_{-\infty}^{+\infty} Z_{t} d w(t) \\
& Z_{\max }=\sup _{t \in(-\infty, \infty)}\left\{Z_{t} w(t)\right\}
\end{aligned}
$$

where $w(t)$ is some weight function.

It is clear that performance of a test statistic generated using Equations (13) and (14) depends on $Z_{t}$ and $w(t)$ which are used for calculating $Z$ and $Z_{\max }$. For $Z_{t}$, two functions are used in the literature. They are given as Pearson statistic and the loglikelihood ratio (LLR) which are defined, respectively, as (Zhang, 2002)

$$
X_{t}^{2}=\frac{N\left\{F_{N}(t)-F_{0}(t)\right\}^{2}}{F_{0}(t)\left\{1-F_{0}(t)\right\}}
$$

and

$$
G_{t}^{2}=2 N\left[F_{N}(t) \log \left\{\frac{F_{N}(t)}{F_{0}(t)}\right\}+\left\{1-F_{N}(t)\right\} \log \left\{\frac{1-F_{N}(t)}{1-F_{0}(t)}\right\}\right] .
$$

It is possible to generate new powerful and distribution symmetric tests by choosing $w(t)$ and $Z_{t}$ functions as presented in Table

1. The resultant test statistics $Z_{K}, Z_{A}$, and $Z_{C}$ are given as (Zhang, 2002) 
$Z_{K}=\max _{1 \leq i \leq N}\left(\left(i-\frac{1}{2}\right) \log \left\{\frac{i-\frac{1}{2}}{N F_{0}\left(X_{(i)}\right)}\right\}+\left(N-i+\frac{1}{2}\right) \log \left[\frac{N-i+\frac{1}{2}}{N\left\{1-F_{0}\left(X_{(i)}\right)\right\}}\right]\right)$

$Z_{A}=-\sum_{i=1}^{N}\left[\frac{\log \left\{F_{0}\left(X_{\left(_{i}\right.}\right)\right\}}{N-i+\frac{1}{2}}+\frac{\log \left\{1-F_{0}\left(X_{\left(^{i}\right.}\right)\right\}}{i-\frac{1}{2}}\right]$

$Z_{C}=\sum_{i=1}^{N}\left[\log \left(F_{0}^{-1}\left(X_{(i)}\right)-1 / \frac{\left(N-\frac{1}{2}\right)}{\left(i-\frac{3}{4}\right)}-1\right)\right]^{2}$.

Table 1. Derivation of new GOF tests using Equations (13) through (16).

\begin{tabular}{|c|c|c|}
\hline $\begin{array}{l}\text { Weight Function } \\
\qquad w(t)\end{array}$ & $\begin{array}{l}\text { Statistic } \\
Z \text { or } Z_{\max }\end{array}$ & $\begin{array}{c}\text { Name of } \\
\text { obtained GOF } \\
\text { Test }\end{array}$ \\
\hline$w(t)=1$ & $\begin{aligned} Z_{\max } & =\sup _{t \in(-\infty, \infty)}\left\{Z_{t} w(t)\right\} \\
Z_{t} & =G_{t}^{2}=2 N\left[F_{N}(t) \log \left\{\frac{F_{N}(t)}{F_{0}(t)}\right\}\right. \\
& \left.+\left\{1-F_{N}(t)\right\} \log \left\{\frac{1-F_{N}(t)}{1-F_{0}(t)}\right\}\right]\end{aligned}$ & $\mathrm{Z}_{\mathrm{K}}$ \\
\hline$d w(t)=F_{N}(t)^{-1}\left\{1-F_{N}(t)\right\}^{-1} d F_{N}(t)$ & $\begin{aligned} Z=\int_{-\infty}^{+\infty} Z_{t} d w(t) & \\
Z_{t}= & G_{t}^{2}=2 N\left[F_{N}(t) \log \left\{\frac{F_{N}(t)}{F_{0}(t)}\right\}\right. \\
& +\left\{1-F_{N}(t)\right\} \log \left\{\frac{1-F_{N}(t)}{1-F_{0}(t)}\right.\end{aligned}$ & $\mathrm{Z}_{\mathrm{A}}$ \\
\hline$d w(t)=F_{0}(t)^{-1}\left\{1-F_{0}(t)\right\}^{-1} d F_{0}(t)$ & $\begin{aligned} Z= & \int_{-\infty}^{\infty} Z_{t} d w(t) \\
Z_{t}= & G_{t}^{2}=2 N\left[F_{N}(t) \log \left\{\frac{F_{N}(t)}{F_{0}(t)}\right]\right. \\
& +\left\{1-F_{N}(t)\right\} \log \left\{\frac{1-F_{N}(t)}{1-F_{0}(t)}\right\}\end{aligned}$ & $\mathrm{Z}_{\mathrm{C}}$ \\
\hline
\end{tabular}

Since the test statistics $Z_{K}, Z_{A}$, and $Z_{C}$ use the likelihood ratio, they are alternatively named as LLR based GOF tests. 


\subsection{OS based GOF Testing}

OS based GOF testing uses the quantiles of the observed data to measure the degree of fit of a distribution to data. In this technique, decision is taken by using the $\rho$-vector of the data (Rostami et al., 2012). $\rho$-vector is considered in several studies on OS based GOF tests (Glen et al., 2001). Thus, calculation of $\rho$-vector is very crucial in OS based spectrum sensing.

Computation of the $\rho$-vector can be carried out using the following steps (Rostami et al., 2012):

- Step \#1 - Transformation: Elements of the received signal vector, $\mathbf{x}$, are transformed via the known noise CDF, $F_{0}(x)$, as

$z_{i}=F_{0}\left(x_{i}\right), \quad i=1,2, \ldots, N$

Then, a new vector $z=\left[z_{1}, z_{2}, \ldots, z_{N}\right]^{T}$ is defined.

- Step \#2 - Sorting: Elements of $z$ are arranged in ascending order of magnitude as $z_{(1)} \leq z_{(2)} \leq \ldots \leq z_{(N)}$. The sorted vector $\tilde{z}=\left[z_{(1)}, z_{(2)}, \ldots, z_{(N)}\right]^{T}$ is formed.

- Step \#3 - Beta Transformation: By transforming the elements of $\tilde{z}$ using Beta CDF, elements of $\rho$-vector are obtained as $\rho_{i}=F_{\beta}\left(z_{(i)} ; i, N-i+1\right)$. Thus, $\rho$-vector is formed as $\rho=\left[\rho_{1}, \rho_{2}, \ldots, \rho_{N}\right]^{T}$. Here, $F_{\beta}(y ; \alpha, \beta)$ denotes the Beta CDF with shape parameters $\alpha$ and $\beta$

After this step has been completed, various test statistics based on $\rho$-vector can be defined. For example, two candidate test statistics can be given as (Glen et al., 2001)

$$
T_{o s}^{1}=\sum_{i=1}^{N}\left|\rho_{i}-0.5\right| \text { and } T_{o s}^{2}=\sum_{i=1}^{N}\left(\rho_{i}-0.5\right)^{2}
$$

Also in (Rostami et al., 2012), the authors state that, based on simulations, they could obtain the maximum probability of detection using the following test statistic

$$
T_{O S}^{3}=\sum_{i=1}^{N}\left|\rho_{(i)}-\frac{i}{(N+1)^{2}}\right|
$$

where $\rho_{(i)}$ is the $i^{\text {th }}$ element of the sorted $\rho$-vector (in ascending order).

In the end, a decision is taken by comparing the test statistic with a pre-determined threshold, $\gamma_{o s}$, as in other GOF test statistics. This can be expressed as

$$
\begin{aligned}
& H_{0}: T_{o S} \leq \gamma_{o S}, \text { channel is idle, } \\
& H_{1}: T_{O S}>\gamma_{o S}, \text { channel is busy. }
\end{aligned}
$$

\section{SIMULATION RESULTS}

In this section, the results of the simulations carried out using the models given in Eqs. (1), (2), and (3) are presented. In the simulations, it is assumed that the PU signal is given as a flat or DC valued signal such that $s=\sqrt{\eta} m$ where $m=1$ and $\eta$ corresponds to signal-to-noise ratio (SNR) (Rostami et al., 2012). The noise variance $\sigma^{2}$ is selected as equal to 1 . 
The PU status change points in the models given in Eqs. (2) and (3) are obtained according to Poisson distribution. Arrival and departure rate, $\lambda_{a}$ and $\lambda_{d}$, respectively, parameters of Poisson distribution, are assigned as $\lambda_{a}=\lambda_{d}=1$ (Beaulieu and Chen, 2010). In addition, the change point values are generated so that they are within the interval $[1, N]$ to ensure that the PU status changes occur in the sensing period.

The number of observed samples are assigned as $N=100$ and SNR is assumed to be equal to $-10 \mathrm{~dB}$. The thresholds for all the considered techniques are determined experimentally using Monte Carlo simulations according to which the data under both hypotheses, $H_{0}$ and $H_{1}$, are generated for $10^{5}$ times.

For each realization, the spectrum sensing techniques considered in this letter have been calculated under both hypotheses. Performances of probability of detection, $\mathrm{P}_{D}$, versus probability of false alarm, $P_{F A}$, have been displayed in Figs. 1 and 2 using receiver operating characteristics (ROC) curves.

The abbreviations in the legends of Figs. 1 and 2 are defined as follows: "AD" for Anderson-Darling test, "KS" for KolmogorovSmirnov test, "CM" for Cramer-von Misses test, "ZK", "ZC", and "ZA" for the LLR based test statistics ZK, ZC, and ZA, respectively, and "OS" for the order statistics based GOF test introduced in (Rostami et al., 2012).

In Fig. 1, it can be seen that all the GOF test based spectrum sensing techniques achieve good performance in terms of probability of detection, $\mathrm{P}_{D}$, when there is no PU status change in the channel. In addition, OS based spectrum sensing technique outperforms other methods for all $P_{F A}$ values.

It is clear from Fig. 1 that when there is a PU status change in the channel, detection performances of all the considered techniques drastically decrease.

In Fig.2, it is assumed that strictly one status and at most two status changes occur in the channel. For the case of at most two status changes, one or two status changes of the PU occur in the channel with the same probability. That is, occurrence probabilities of the models given in Eqs. (2) and (3) are equal.

According to results in Fig. 2, it can be said that the detection performances of the GOF test based spectrum sensing methods worsen as the PU traffic density in the channel increases.

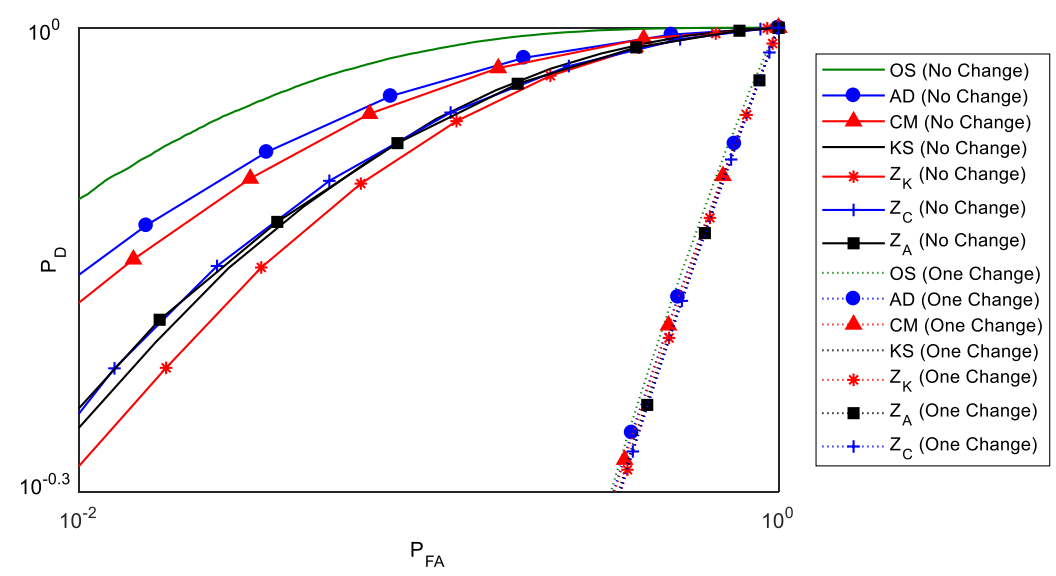

Figure 1. $\mathrm{P}_{D}$ vs. $\mathrm{P}_{F A}$ for no status change and one status change of the PU. 


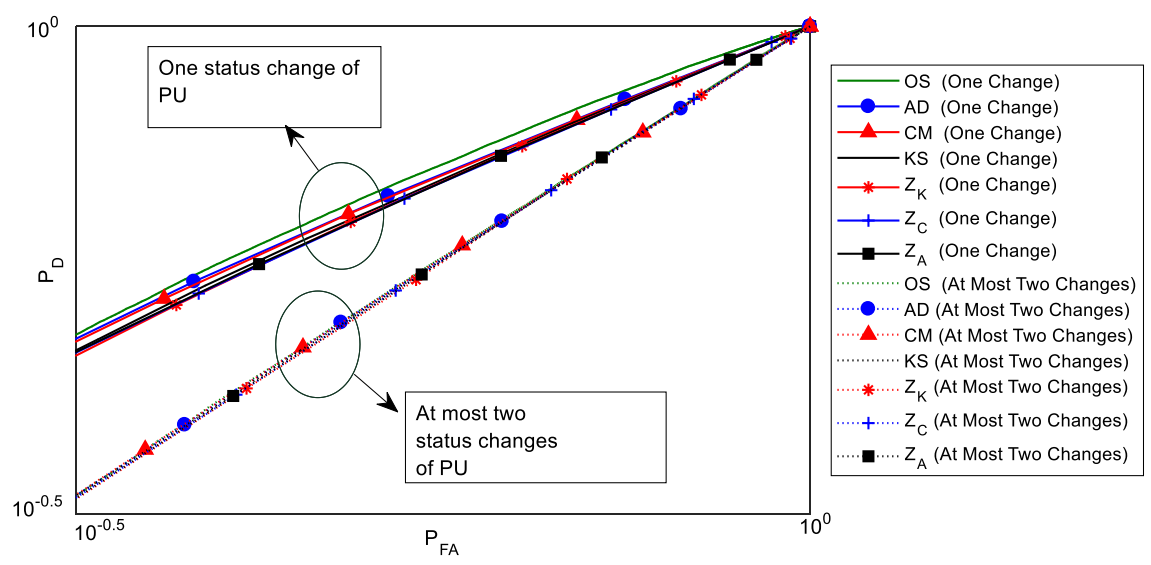

Figure 2. $\mathrm{P}_{D}$ vs. $\mathrm{P}_{F A}$ for one status change and at most two status changes of the PU.

\section{CONCLUSIONS}

In this study, the effect of PU traffic on the performance of GOF test based spectrum sensing methods in CR has been investigated.

Seven different GOF tests have been considered. Simulations have been carried out for the three cases of no status change, one status change, and at most two status changes of the PU in the channel. According to simulation results, it has been observed that PU traffic causes deterioration of the detection performance of the GOF test based spectrum sensing methods.

As a future study direction, we plan to search for more robust GOF test based spectrum sensing techniques in order to increase the probability of detection performance under PU traffic.

\section{REFERENCES}

Arshad, K., \& Moessner, K. (2013). Robust spectrum sensing based on statistical tests. IET Comm., 7 (9), 808- 817. doi: 10.1049/iet-com.2012.0499

Beaulieu N. C., \& Chen Y. (2010). Improved energy detectors for cognitive radios with randomly arriving or departing primary users. IEEE Signal Processing Letters, 17 (10), 867-870. doi: 10.1109/LSP.2010.2064768

Düzenli, T., \& Akay, O. (2013). Bilişsel radyolar için birincil kullanıcı trafiği içeren kanallarda dinamik programlama ve ortalama kümülatif toplama dayalı yeni bir test istatistiğinin önerilmesi. V. İletişim Teknolojileri Ulusal Sempozyumu, İzmir, Turkey, 1-10.

Düzenli, T., \& Akay, O. (2016). A new spectrum sensing strategy for dynamic primary users in cognitive radio. IEEE Communications Letters, 20 (4), 752-755. doi: 10.1109/LCOMM.2016.2527640

Glen, A. G., Leemis, L. M., \& Barr, D. R. (2001). Order statistics in goodness-of-fit testing. IEEE Transactions on Reliability, 50 (2), 209-213. doi: 10.1109/24.963129

Lei, S., Wang, H., \& Shen, L. (2011). Spectrum sensing based on goodness of fit tests. International Conf. on Electr., Comm. and Cont. (ICECC), Ningbo, China, 485 - 489. doi: 10.1109/ICECC.2011.6067691

N.-Thanh, N., K.-Xuan, T., \& Koo, I. (2012). Comments on "Spectrum sensing in cognitive radio using goodness-of-fit testing". IEEE Trans. on Wireless Comm., 11 (10), 3409 - 341. doi: 10.1109/TWC.2012.081312.110951

Rostami, S., Arshad, K., \& Moessner, K. (2012). Order-statistic based spectrum sensing for cognitive radio. IEEE Comm. Lett., 16 (5), 592 -595. doi: 10.1109/LCOMM.2012.030512.111887

Stephens, M. A. (1974). EDF statistics for goodness of fit and some comparisons. Journal of the American Statistical Association, 69 (347), 730-737. 
Wang, H., Yang, E., Zhao, Z., \& Zhang, W. (2009). Spectrum sensing in cognitive radio using goodness of fit testing. IEEE Trans. on Wireless Comm., 8 (11), 5427 - 5430. doi: 10.1109/TWC.2009.081586

Yücek, T., \& Arslan, H. (2009). A survey of spectrum sensing algorithms for cognitive radio applications. IEEE Communications Survey \& Tutorials, 11 (1), 116-130. doi: 10.1109/HMI.2016.7449196

Zhang, J. (2002). Powerful goodness-of-fit tests based on the likelihood ratio. J. R. Statist. Soc. B Part (2), 64, 281- 294. doi: $10.1111 / 1467-9868.00337$ 